\title{
The prevalence of self-reported lower limb and foot health problems experienced by participants with systemic lupus erythematosus: Results of a UK national survey
}

\author{
L Cherry $^{1,2}$, B Alcacer-Pitarch ${ }^{3}$, N Hopkinson ${ }^{4}$, LS Teh ${ }^{5}$, EM Vital ${ }^{3}$, CJ Edwards ${ }^{6}$, A Blake and AE Williams $^{8}$ \\ ${ }^{1}$ Faculty of Health Sciences, University of Southampton, UK; ${ }^{2}$ Department of Podiatry, Solent NHS Trust, UK; ${ }^{3}$ NIHR Leeds Biomedical \\ Research Unit, Leeds Teaching Hospitals NHS Trust and Leeds Institute of Rheumatic and Musculoskeletal Medicine, University of Leeds, UK; \\ ${ }^{4}$ Department of Rheumatology, Royal Bournemouth/Christchurch Hospitals NHS Foundation Trust, UK; ${ }^{5}$ Department of Rheumatology, Royal \\ Blackburn Hospital, UK; ${ }^{6}$ NIHR Wellcome Trust Clinical Research Facility, University Hospital Southampton NHS Foundation Trust, UK; \\ ${ }^{7}$ Private podiatrist, Verwood, UK; and ${ }^{8}$ School of Health Sciences, University of Salford, UK
}

\begin{abstract}
Objective: The main aim of this survey was to determine the frequency of self-reported lower limb or foot and ankle complications experienced by participants with systemic lupus erythematosus (SLE). A secondary aim was to determine the frequency of treatments that have been received or that participants with SLE may like to receive if offered. Method: A quantitative, cross-sectional, self-reported survey design was utilized. The developed survey was checked for face and content validity prior to patient partner cognitive debriefing in order to ensure usability, understanding of the process of completion and of the questions posed. The full protocol for survey development has been published previously. Results: This is the first comprehensive national UK survey of lower limb and foot health problems reported by participants with SLE. A high prevalence of vascular, dermatological and musculoskeletal complications was reported by survey respondents. Additionally, whilst the relative prevalence of sensory loss was low, a quarter of people reported having had a fall related to changes in foot sensation demonstrating a previously unknown rate and cause of falls. Conclusion: Complications related to vascular, dermatological and musculoskeletal health are identified as particularly prevalent in participants with SLE. Further, there is a suggestion that the provision of interventions to maintain lower limb health is highly varied and lacks national standardization, despite there being a strong indication of participant reported need. The findings of this work can be used to inform care guideline development in addition to identifying areas for future research. Lupus (2016) $\mathbf{0}, 1-7$.
\end{abstract}

Key words: Systemic lupus erythematosus; foot; pain; mobility; infection; survey

\section{Background}

Systemic lupus erythematosus (SLE) is a complex, chronic, multi-system autoimmune disease which varies in prevalence and incidence depending upon ethnicity and is diagnosed about nine times more frequently in women than men. ${ }^{1,2}$ In the UK approximately 25,000 people have a diagnosis of SLE. ${ }^{3}$ The disease is highly heterogeneous in that

Correspondence to: Lindsey Cherry, University of Southampton, Faculty of Health Sciences, Building 45, Burgess Road, Southampton, SO17 1BJ, UK.

Email: 1.cherry@soton.ac.uk

Received 6 June 2016; accepted 16 August 2016

(c) The Author(s), 2016. Reprints and permissions: http://www.sagepub.co.uk/journalsPermissions.nav it varies in clinical and serological manifestations and severity between individuals. Disfigurement caused by the involvement of skin and joints ${ }^{3}$ can affect the patient's perception of body image and sexuality and can have an impact on emotional health $^{4}$ and overall quality of life. ${ }^{5}$

Involvement of the musculoskeletal system is common during the clinical course of SLE, occurring in up to $95 \%$ of patients, with joint pain being the first presenting symptom in up to $50 \%$ of cases. ${ }^{6}$ The extra-articular manifestations of SLE include soft tissue pathology such as capsular swelling, synovial hypertrophy (thickening of joint lining) and tenosynovitis, ${ }^{7}$ which can lead to tendon rupture ${ }^{8}$ or tendon contracture. ${ }^{9}$ Tendons are the force transmitting units of 
the musculoskeletal system, but due to their low metabolic rate and slow healing, injury can result in considerable morbidity and prolonged disability. ${ }^{10,11}$ In relation to the symptoms associated with muscle involvement, these can range in severity from mild aches in up to $80 \%$ of cases to painful inflammatory myositis in up to $11 \%$ of cases. ${ }^{7}$

In addition to the musculoskeletal involvement in cases of SLE, people may also present with vasculopathy. Vascular involvement can be either a direct complication of SLE or develop as comorbidity and represents one of the most frequent causes of death. ${ }^{12}$ Mathieu et al. ${ }^{13}$ identified that abnormal vascular reactivity and coagulopathy both contribute to an increased risk of atheroma and therefore recommend careful monitoring for any vascular change with the aim of preventing tissue necrosis and ultimately amputation or death.

Focussing on the foot, a narrative review of the literature ${ }^{14}$ identified that SLE affects foot and lower limb morbidity and that these have the potential to impact upon health related quality of life. However, the scale of these problems is unclear and little research in this area has been completed to date. ${ }^{15}$ It could be speculated that the altered musculoskeletal, peripheral neurovascular and tissue health experienced by people with SLE renders their feet particularly susceptible to deformity, poor function, ulceration and/or infection and pain. However the effects and extent of SLE upon body systems within the periphery are unclear; e.g. with consideration to neurological function, the extent of patient reported motor neuropathy (e.g. muscle weakness), sensory neuropathy (e.g. lost pressure detection) or autonomic neuropathy (e.g. poor temperature regulation) is unclear. Further, the attention given to patients' feet during the clinical consultation may be limited, as has been evidenced for people with rheumatoid arthritis. ${ }^{16}$ Thus, the opportunity to provide timely advice and clinical intervention is missed. As such, there is a need to determine the prevalence of lower limb or foot and ankle complications experienced by people with SLE. Thus, the main aim of this survey was to determine the frequency of self-reported lower limb or foot and ankle complications experienced by people with SLE. A secondary aim was to determine the frequency of treatments that have been received or that people with SLE may like to receive if offered.

\section{Methods}

\section{Ethics, consent and permissions}

Ethical approval for the study was granted by the University of Salford (HSCR14/25) and NRES (14/SC/1009). All research was completed in accordance with the Declaration of Helsinki guidelines for research practice. Information about the purpose and intended use of the study was included in a covering letter as part of the survey data sheet. Consent to take part in the survey was considered implicit following the completion and return of the form.

\section{Study and survey design}

A quantitative, cross-sectional, self-reported survey design was utilized. To aid survey design a patient and practitioner advisory group was formed. The group was comprised of members of the research team, rheumatologists, podiatrists and participants with SLE. An initial group meeting, held following review of existing literature related to problems associated with SLE, generated an item pool from which the survey could be developed. From this preliminary work, the survey was developed based on the agreed themes, categories, question format and overall structure. ${ }^{17}$ The survey was checked for face and content validity prior to cognitive debriefing in order to ensure usability, understanding of the process of completion and of the questions posed. The choice of language within the survey was considered and advice sought during the survey development process from the patient partners. Where it was felt that the use of technical terminology was appropriate, clarifying text was also included; e.g. 'Have you had any breaks in the skin that have been slow to heal (known as ulceration)?'. The full protocol for survey development has been published previously. ${ }^{17}$

The final survey consisted of five sections: 1. Lower limb circulatory health; 2. Lower limb nerve function; 3. Foot and ankle skin health; 4. Lower limb musculoskeletal health; and 5. Received or 'needed' foot and ankle treatments.

\section{Survey distribution}

The survey was distributed consecutively to all eligible participants with SLE attending six UK NHS Rheumatology departments, with whom the research team are associated (Blackburn, Christchurch, Leeds, Manchester, Salford and Southampton), and distributed to all members 
of the Lupus UK membership register. Using this approach, it was not possible to determine how many people declined to participate in the survey.

\section{Analysis}

All analysis was completed using SPSS version 18.0 (Chicago, USA). Prior to analysis, data were checked for inconsistencies, outliers or missing information. Descriptive statistics (count and percentage data) were used throughout.

\section{Results}

\section{Survey data}

A total of 182 completed surveys were returned to the research team. The reported age range of participants was normally distributed, with the majority of participants being within the ranges of 40-49 years and 50-59 years. The mean reported disease duration was $14.6( \pm 9.9)$ years. Nineteen participants were active smokers. The reported ethnicity of participants was as follows: white $n=125$, black Caribbean $n=3$, black African $n=3$, black other $n=3$, Indian $n=7$, Pakistani $n=7$, Bangladeshi $n=1$, Chinese $n=6$, other $n=4$, non-disclosed $n=23$.

\section{Lower limb circulatory health}

Overall, $85 \%(n=156)$ reported having experienced some form of lower limb circulatory impairment. As shown in Table 1, the most frequently reported symptom of impaired circulation was night cramps $(74 \% ; n=135)$ and the least frequent was chilblains $(21 \% ; n=38)$.

The receipt of treatment for lower limb circulatory complications was reported by $18 \%(n=32)$. Reported treatments included prescribed medication use $66 \%(n=21)$, surgical intervention $19 \%$ $(n=6)$, or compression hosiery $6 \%(n=2)$. The nature of treatment was unspecified by three people. The reported prescribed medications included Nifedipine, Clopidogrel, Sildenafil, Cyclophosphamide, Iloprost or Epoprostenol Sodium.

\section{Lower limb nerve function}

Overall, 16\% $(n=29)$ reported having experienced symptoms consistent with sensory neuropathy (described as a 'loss of feeling' for the purposes of this survey), whilst $25 \%(n=46)$ reported having
Table 1: Reported prevalence of self-reported lower limb complications experienced by people with systemic lupus erythematosus

\begin{tabular}{|c|c|c|}
\hline & Ever $\mathrm{n}(\%)$ & Currently $\mathrm{n}(\%)$ \\
\hline \multicolumn{3}{|l|}{ Circulation } \\
\hline Raynaud's & $93(51)$ & $34(19)$ \\
\hline Chilblains & $38(21)$ & $14(8)$ \\
\hline Intermittent claudication & $99(54)$ & $30(16)$ \\
\hline Night cramps & $135(74)$ & $33(18)$ \\
\hline \multicolumn{3}{|l|}{ Neurological } \\
\hline Sensory loss & $29(16)$ & $12(7)$ \\
\hline Sensory loss related fall & $46(25)$ & - \\
\hline \multicolumn{3}{|l|}{ Skin health } \\
\hline Ulceration & $44(24)$ & $15(8)$ \\
\hline Callus or corns & $129(71)$ & $75(41)$ \\
\hline Onychocryptosis & 68 (37) & $23(13)$ \\
\hline Rashes or blistering & $62(34)$ & $11(6)$ \\
\hline \multicolumn{3}{|l|}{ Skin infection } \\
\hline Tinea pedis & $76(42)$ & $20(11)$ \\
\hline Onychomycosis & $65(36)$ & $35(19)$ \\
\hline Verrucae pedis & $76(42)$ & $16(9)$ \\
\hline \multicolumn{3}{|l|}{ Musculoskeletal health } \\
\hline Joint pain & $145(80)$ & $60(33)$ \\
\hline Joint stiffness & $134(74)$ & $50(27)$ \\
\hline Joint swelling & $93(51)$ & $29(16)$ \\
\hline Change in foot shape & $65(36)$ & - \\
\hline Functional impairment & $67(37)$ & $22(12)$ \\
\hline
\end{tabular}

had a fall because of altered sensation in their feet. Whilst specific questions relating to painful neuropathy were not included in the survey, 10 patients reported experiencing symptoms consistent with neuropathic pain within the free text comments section of the survey.

The receipt of treatment for lower limb nerve complications was reported by $8 \% \quad(n=14)$. Reported treatments included Amitriptyline or Pregabalin, steroid injection, Extra-Corporeal Shockwave Therapy, Low Level Laser Therapy or Acupuncture.

\section{Foot and ankle skin health}

Overall, $86 \%(n=157)$ reported having experienced some form of foot or ankle skin health complaint. As shown in Table 1, the most frequently reported complication was callus or corn formation $(71 \%$; $n=129)$ and the least frequent was ulceration $(24 \% ; n=44)$.

'Having ever' experienced viral skin infection (verrucae pedis) was reported by $42 \% \quad(n=77)$, fungal skin infection by $42 \%(n=76)$ and fungal nail infection (onychomycosis) by $36 \% \quad(n=66)$. Comparatively, reported previous or current bacterial infection, for which antibiotic treatment was 
issued, was lowest at 15\% $(n=27)$ and $2 \%(n=3)$ respectively.

\section{Lower limb musculoskeletal health}

Overall, $158(87 \%)$ reported having experienced some form of lower limb musculoskeletal complication. As shown in Table 1, the most frequently reported complication was joint pain $(80 \%$; $n=145)$ and the least frequent was a change in foot shape $(36 \% ; n=65)$. A previous or current episode of loss of function, such that it was difficult or impossible to walk, was reported by $37 \%$ $(n=67)$ and $12 \%(n=22)$ respectively.

\section{Received or wanted foot and ankle treatments}

Overall, 33\% $(n=60)$ reported having ever discussed their foot health with a doctor (rheumatologist or general practitioner) and $23 \% \quad(n=41)$ reported having ever been diagnosed with a specific foot or ankle problem.

A summary of the frequency of the common treatments reportedly received is shown in Table 2. The number of participants who reported that they thought they would benefit from these treatments is also shown. A further $27 \%(n=50)$ of participants additionally reported that they thought they would benefit from general foot health and care advice.

\section{Discussion}

To our knowledge, this is the first comprehensive national UK survey of lower limb and foot health problems reported by people with SLE. This novel survey has captured the reported high prevalence of vascular, dermatological and musculoskeletal

Table 2: Treatment received and "would like to receive" to aid foot health people with systemic lupus erythematosus

\begin{tabular}{llll}
\hline & $\begin{array}{l}\text { Received } \\
(\text { NHS })\end{array}$ & $\begin{array}{l}\text { Received } \\
\text { Private) } \\
\mathrm{n}(\%)\end{array}$ & $\begin{array}{l}\text { Would like } \\
\text { to receive } \\
\mathrm{n}(\%)\end{array}$ \\
\hline $\begin{array}{l}\text { Oreatment } \\
\text { Specialist footwear }\end{array}$ & $\begin{array}{l}49(27) \\
\text { Footwear advice }\end{array}$ & $10(5)$ & $51(28)$ \\
Removal of callus & $35(19)$ & $4(2)$ & $19(10)$ \\
$\quad 20(11)$ & $8(4)$ & $49(27)$ \\
Nail care & $18(10)$ & $13(7)$ & $49(27)$ \\
Nail surgery & $12(7)$ & $3(2)$ & $54(30)$ \\
Surgery & $28(15)$ & 0 & $9(5)$ \\
\hline
\end{tabular}

complications experienced by this group of participants. Additionally, whilst the relative prevalence of sensory loss was low, a quarter of participants reported having had a fall related to changes in foot sensation. This demonstrates a previously unknown potential cause of falls amongst people with SLE. Awareness of potential falls risk factors creates opportunity to target prevention strategies. Importantly, this patient population is already at increased fracture risk due to comorbid health needs and/or treatments and therefore there is a potentially large detrimental consequence of falling.

Interestingly, the findings of this UK survey are similar to those reported by Otter et al. ${ }^{18}$ in a crosssectional study completed in New Zealand. The comparative reported prevalence of vascular, musculoskeletal and neurological complications are similar between studies suggesting that, despite differences in methodological approach, the findings may on average accurately reflect the foot health status of this group of participants. However to date, to our knowledge, there is currently no comparative research available against which it is possible to compare the prevalence of infection (approximately half of participants) or callus/corns (approximately three-quarters of participants) identified in this survey.

There was a high prevalence of vascular complications and symptoms, with varied drug or surgical intervention, reported. Of note, $85 \%$ of participants reported having experienced some form of vascular complication whilst only $18 \%$ of participants reported having received treatment for peripheral vascular health. The results of this survey therefore suggest a current mismatch between participant reported vascular symptoms and treatment. Additionally, a range in treatment approaches were reported. Overall, the authors suggest that the findings of this survey show a potential area for improved clinician and patient awareness and standardization of clinical practice (e.g. identifying when prophylactic pharmacology, such as Sildenafil, is indicated). It is hypothesized by the research team that this could be achieved nationally through guidance documentation or locally through advice and screening at each consultation in order to detect and treat early changes and reduce the risk of serious progressive consequences such as ulceration and necrosis. ${ }^{19}$ Although not evidenced by the findings of this study, arguably, there is potential for non-drug treatment approaches to also improve lower limb vascular health (e.g. exercise for the reduction of intermittent claudication symptoms), although further 
research is required to determine the mobility impact of SLE and to evaluate the potential efficacy of exercise/mobility prescription in participants with SLE. ${ }^{20}$

Participants did note experiencing loss of feeling, with symptoms of 'numbness' and/or neuropathic pain also referenced in the free text comments received. Additionally, a number of participants reported falls as a consequence of altered sensation in their feet. However, there is insufficient detail in the context of this survey to further clarify the exact nature of the altered sensation experienced and how this led to a fall; or indeed whether the fall related specifically to factors directly related to SLE. Further qualitative research that clarifies the nature and impact of such problems experienced by this group is warranted. It is also proposed that mobility and falls may represent an important and potentially under-appreciated area of concern that requires further investigation.

Over a third of participants reported having ever experienced an episode of musculoskeletal complication that resulted in an inability to walk. This has a high potential for reduced mobility and subsequently may have far reaching impact on comorbid health complications (e.g. further compromise of vascular status or compound tissue deconditioning) which does not appear to be addressed by current health care practice for this population. There is a need to further explore the impact of compromised mobility experienced by this patient group on health, work, socialization or quality of life.

Foot problems that are prevalent in the general population such as corns, callus and nail thickening were also evident in this group. However, it is theoretically possible that the consequences of these problems can be progressive and multifactorial, leading quickly to tissue breakdown, ulceration with potential for resistant bacterial infection or necrosis and delayed wound healing. In addition, fungal and viral infections may become more widespread due to the autoimmune nature of SLE and the immunosuppressive medication that can be used for its management. It is recommended that further research is required to fully appreciate the nature of infection prevalence, identification and risk management/treatment in this patient group. The impact and burden of self-treatment for repeated fungal infections is reported in free text responses and a need for professional diagnosis and advice about the most effective treatments suggested.

Despite the seemingly high prevalence of lower limb complications reported, only a third of respondents reported ever having been asked about their foot health by a doctor and even fewer reported having been given any specific diagnosis. However, it is possible that the patients do not report these problems to a doctor, as talking about feet may not be seen as a medical role; this lack of disclosure is reported by Blake et al. ${ }^{21}$ in their study of patients with rheumatoid arthritis. It is currently unclear whether this is also the case for people with SLE.

The most frequent intervention for foot problems reported in this survey was the provision of foot orthoses. However, despite being the most frequent intervention, this was only reported by $27 \%$ of participants and a clear request for further assistance was evidenced, especially for general foot health education or nail and skin care. However, further research is needed to evaluate the effectiveness of the range of potential foot health interventions for this patient group. It is perhaps of note that the number of participants reporting that they would like to receive treatment is greater than those who have had treatment of any kind. This represents a potentially unmet foot health care burden and further research to better understand the specific nature of any unmet need is required.

There may be unknown responder bias evidenced within the findings of this survey and this represents a limitation of this work; it may be that those participants who have previously experienced complications are more likely to complete and return the survey. The results of the survey may also be subject to recall bias; e.g. participants may over or under-report phenomena depending upon their ability to accurately recall their past experiences. Further the survey was limited to a UK population although data were collected from varied geographical locations nationally. Globally, SLE is demographically variable and there is the need to replicate this survey in other global contexts to fully appreciate the ranging prevalence and burden of poor foot health for people with SLE. Due to the self-reported nature of the survey, it was not possible to ascertain the participants' current SLE disease activity. The findings of this initial survey could therefore be enhanced with the completion of disease activity and damage indices through additional clinical study.

Despite the limitations, this is the first UK survey to obtain data about the prevalence and management of self-reported foot problems experienced by participants with SLE. The findings indicate that foot health should be part of a health care consultation. A particular focus on vascular, dermatological and musculoskeletal complications is 
indicated although neurological health and falls risk should also be considered.

\section{Conclusion}

This novel national survey demonstrates a high prevalence of previously unreported lower limb and foot health complications experienced by participants with SLE and therefore achieves the primary research aim. Complications related to vascular, dermatological and musculoskeletal health are identified as particularly prevalent in participants with SLE. The frequency of treatments reported was relatively low compared to the reported prevalence of complication or reported perceived need. The type of treatments currently offered are variable and appear to lack national standardization. Overall, there is a high prevalence of lower limb complications reported by participants with SLE for which treatment is currently reported as nationally variable or not offered, although is perceived as needed by the majority.

\section{Acknowledgements}

We thank Kevin Kennard and Helen Bifield as patient partners and the Logres Trust who provided the funding for this study. The publication plan was agreed with the trustees of the Logres Trust. The research is supported by the National Institute for Health Research (NIHR) Leeds Musculoskeletal Biomedical Research Unit. The views expressed are those of the authors and not necessarily those of the NHS, the NIHR or the Department of Health. The author's contributions were as follows: LC and AW led the design of the study, the coordination of the study and LC led the preparation of data, completion of analysis and drafting of the manuscript; $\mathrm{CE}, \mathrm{NH}, \mathrm{BAP}, \mathrm{AB}$ and EV participated in the protocol development and LST contributed to the inception of the study; all authors contributed to, read and have approved the final manuscript.

\section{Declaration of Conflicting Interests}

The author(s) declared no potential conflicts of interest with respect to the research, authorship, and/or publication of this article.

\section{Funding}

The author(s) disclosed receipt of the following financial support for the research, authorship, and/or publication of this article: Drs Anita Williams and Lindsey Cherry received a grant to undertake this work from the Logres Trust, a UK charity that funds research into Rheumatic diseases. Dr L Cherry was supported in her role by a UK National Institute for Health Research Clinical Lecturer Fellowship.

\section{References}

1 Lau CS, Yin G, Mok MY. Ethnic and geographical differences in systemic lupus erythematosus: an overview. Lupus 2006; 15 : 715-719.

2 D'Cruz DP, Khamashta MA, Hughes GR. Systemic lupus erythematosus. Lancet 2007; 369: 587-596.

3 Nightingale AL, Farmer RD, de Vries CS. Systemic lupus erythematosus prevalence in the UK: methodological issues when using the General Practice Research Database to estimate frequency of chronic relapsing-remitting disease. Pharmacoepidemiol Drug Saf 2007; 16: 144-151.

4 Hale ED, Treharne GJ, Norton Y, et al. 'Concealing the evidence': the importance of appearance concerns for patients with systemic lupus erythematosus. Lupus 2006; 15: 532-540.

5 McElhone K, Abbott J, Gray J, Williams A, Teh LS. Patient perspective of systemic lupus erythematosus in relation to healthrelated quality of life concepts: a qualitative study. Lupus 2010; 19: $1640-1647$.

6 Griffiths B, Mosca M, Gordon C. Assessment of patients with systemic lupus erythematosus and the use of lupus disease activity indices. Best Pract Res, Clin Rheumatol 2005; 19: 685-708.

7 Petri M. Musculoskeletal complications of systemic lupus erythematosus in the Hopkins Lupus Cohort: an update. Arthritis Care Res 1995; 8: 137-145.

8 Zoma A. Musculoskeletal involvement in systemic lupus erythematosus. Lupus 2004; 13: 851-853.

9 Ostendorf B, Scherer A, Specker C, Mödder U, Schneider M. Jaccoud's arthropathy in systemic lupus erythematosus: differentiation of deforming and erosive patterns by magnetic resonance imaging. Arthritis Rheum 2003; 48: 157-165.

10 Petri M. Musculoskeletal complications of systemic lupus erythematosus in the Hopkins Lupus Cohort: an update. Arthritis Care Res 1995; 8: 137-145.

11 Grigor R, Edmonds J, Lewkonia R, Bresnihan B, Hughes GR. Systemic lupus erythematosus. A prospective analysis. Ann Rheum Dis 1978; 37: 121-128.

12 Bernatsky S, Boivin JF, Joseph L, et al. Mortality in systemic lupus erythematosus. Arthritis Rheum 2006; 54: 2550-2557.

13 Mathieu A, Sanna G, Passiu G, Cauli A, Piga M. Raynaud's syndrome in systemic lupus erythematosus. Lancet 2000; 355: 1102-1103.

14 Williams AE, Crofts G, Teh LS. 'Focus on feet'-The effects of systemic lupus erythematosus: a narrative review of the literature. Lupus 2013; 22: 1017-1023.

15 Williams AE, Graham AS. 'My feet: visible, but ignored...' A qualitative study of foot care for people with rheumatoid arthritis. Clin Rehabil 2012; 26: 952-959.

16 Oppenheim AN. Questionnaire Design, Interviewing and Attitude Measurement. New York: Bloomsbury Publishing, 2001.

17 Williams AE, Cherry L, Blake A, et al. An Investigation into the Scale and Impact of Self-Reported Foot Problems Associated with 
Systemic Lupus Erythematosus: A Study Protocol and Survey Questionnaire Development. Musculoskeletal Care 2016; 14: 110-115.

18 Otter SJ, Kumar S, Gow P, et al. Patterns of foot complaints in systemic lupus erythematosus: a cross-sectional survey. $J$ Foot Ankle Res 2016; 9: 10.

19 Albrecht J, Berlin JA, Braverman IM, et al. Dermatology position paper on the revision of the 1982 ACR criteria for systemic lupus erythematosus. Lupus 2004; 13: 839-849.
20 Lane R, Ellis B, Watson L, Leng GC. Exercise for intermittent claudication. Cochrane Database Syst Rev 2014; 7.

21 Blake A, Mandy PJ, Stew G. Factors influencing the patient with rheumatoid arthritis in their decision to seek podiatry. Musculoskeletal Care 2013; 11: 218-228. 